\title{
Die Abdeckerei und die Hinrichtungsstätte in Kamienna Góra (Landeshut) und Złotoryja (Goldberg), Polen. Zwei Beispiele dargestellt an Hand archäologischer und historischer Forschungen
}

\author{
Paweł Duma $^{1}$ - Daniel Wojtucki ${ }^{2}$ \\ 1 Institut für Archäologie, Universität zu Wrocław/Breslau, ul. Szewska 48, 50-139 Wrocław, Polen, \\ ORCID nr 0000-0001-7991-3133 \\ 2 Institut für Geschichte, Universität zu Wrocław/Breslau, ul. Szewska 49, 50-139 Wrocław, Polen \\ ORCID nr 0000-0002-8539-4410
}

Received $16^{\text {th }}$ November 2018; accepted $13^{\text {th }}$ April 2019

\begin{abstract}
RASOVNY A POPRAVIŠTĚ V KAMENNÉ HOŘE (LANDESHUT) A ZLOTORYJI (GOLDBERG) V POLSKU. DVA PŘÍKLADY UKAZUJÍCÍ VÝSLEDKY ARCHEOLOGICKÝCH A HISTORICKÝCH VÝZKUMŮ
\end{abstract}

\begin{abstract}
ABSTRAKT Cílem př́spěvku je představení výsledků výzkumů, které byly prováděny na bývalých popravištích v Kamenné Hoře a Zlotoryji (Dolní Slezsko, Polsko). V průběhu archeologických výzkumů v obou lokalitách byly nalezeny pozůstatky zděných šibenic o průměru od 5,2 do $7,5 \mathrm{~m}$ a také soubor různých kosterních částí. Ty byly předány $\mathrm{k}$ antropologické a archeozoologické analýze. Nejvíce kostí bylo objeveno ve velké jámě u šibenice v Kamenné Hoře. V př́ípadě Zlotoryji se nejvíce pozůstatků nacházelo uprostřed konstrukce. Během prací nebyly ani na jednom stanovišti nalezeny anatomické hroby přesto, že o ukládání těl na těchto místech informovaly písemné zdroje. Po ukončení výzkumů se započalo s bádáním v historických archívech. Současně analýza kostí ukázala, že jak kosti z vnitř̌ku šibenice tak i z okolí patří hlavně zviŕatům. V prŕípadě Kamenné Hory bylo lidských pozůstatků méně než $5 \%$ z celého souboru, v př́padě Zlotoryji méně než $6 \%$. Celkově dominovaly kosti koní, psů, koček a skotu. Výzkumy ukázaly velmi důležitou sanitární funkci bývalých popravišt', která byla $\mathrm{v}$ mnoha městech využívána k zakopávání padlých zvířat z okolních obcí.
\end{abstract}

KLÍČOVÁ SLOVA šibenice; popraviště; Slezsko; období novověku

ABSTRACT The aim of the paper is to present the results of the research that was carried out at the former execution sites in Kamienna Góra and Złotoryja (Lower Silesia, Poland). During the archaeological excavations in both localities, there were found remains of brick gallows with a diameter of 5.2 to $7.5 \mathrm{~m}$ and also a set of skeletal parts. These were passed on to anthropological and archeozoological analysis. Most bones were found in a large pit near the gallows in Kamienna Góra. In the case of Złotoryja, the most remains were in the middle of the structure. Even during the work, anatomical graves were not found at one site, despite the fact that written sources informed about the storage of these bodies. After the excavations, research in historical archives began. At the same time, bone analysis showed that bones from the inside of the gallows and the surrounding area belong mainly to animals. In the case of Kamienna Góra, human remains were less than 5\% of the entire colection and less than $6 \%$ in the case of Złotoryja. Overall, the bones of horses, dogs, cats and cattle dominated. Research has shown a very important sanitary function of former scaffolds, which has been used in many cities to burrow fallen animals from nearby communities.

KEY WORDS Gallows; Scaffold; Silesia; Modern era

\section{VORWORT}

Den Ort, an dem der Scharfrichter oder seine Knechte die Kadaver, Abfälle, Selbstmörder und manchmal auchVerurteilte vergraben haben, nannte man früher Schinderplatz, Schinderanger oder Schinderwiese. Nicht weit davon entfernt befand sich auch die Schindergrube.Traditionell und aufgrund hygienischer Gegebenheiten, liegen diese Plätze oft in der 


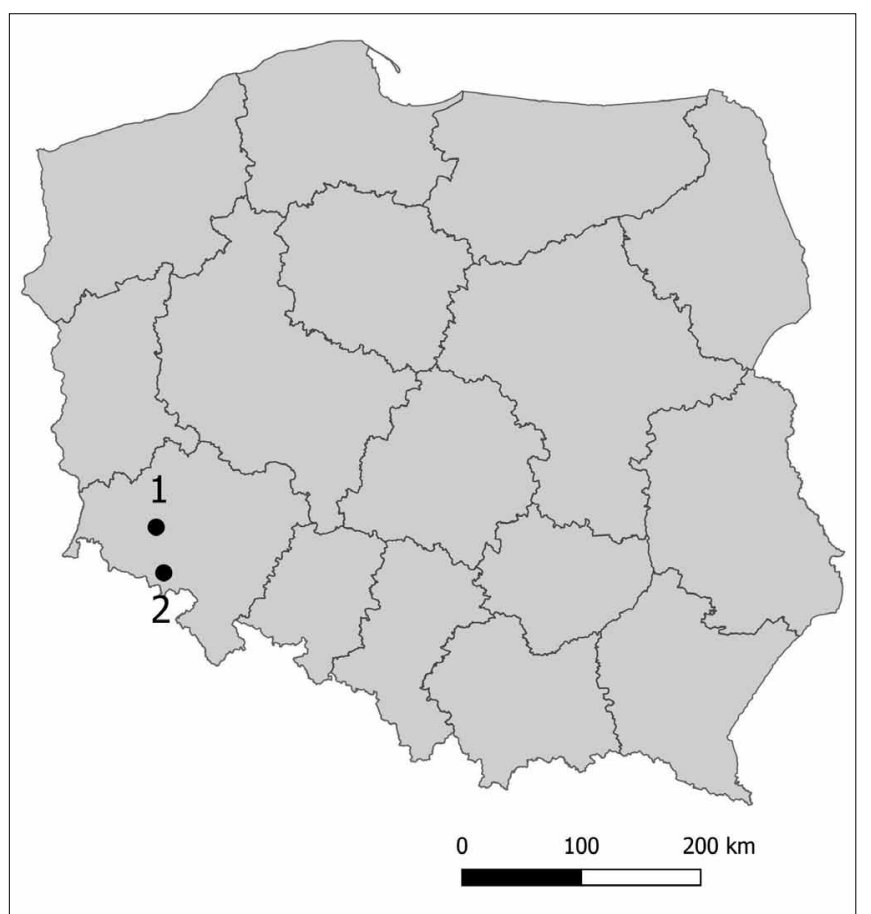

Figur 1. Złotoryja/Goldberg (1) und Kamienna Góra/Landeshut (2) Lokalisation des Ortes im heutigen Polen. Bearbeitung: P. Duma

Nähe von Galgen und anderen Hinrichtungsplätzen. Mit der Größe des Ortes stieg die Zahl dieser Plätze. Dank der in den letzten Jahren durchgeführten archäologischen Forschungen, die vom National Science Center (NCN 2013/11/D/ HS3/02478) finanziert wurden, konnten zwei ehemalige Hinrichtungsstätten erforscht werden, die beide in unmittelbarer Nähe zu einer Abdeckerei lagen. Die Anlagen befanden sich in Kamienna Góra (Landeshut) und in Złotoryja (Goldberg) in Niederschlesien (Polen) (Fig. 1). Die Existenz der Abdeckerei konnte durch Abgleich mit historischen Quellen bestätigt werden. Während der archäologischen Forschungen wurden zahlreiche Tierknochen und Kleinstobjekte entdeckt. Sie ermöglichen, die Praktiken der Scharfrichter und Abdecker teilweise zu rekonstruieren und gehören damit zu jenen seltenen Beispielen dieser Art.

\section{DIE ABDECKEREI UND DER HINRICHTUNGSPLATZ IN KAMIENNA GÓRA (LANDESHUT)}

Der Hinrichtungsplatz in Kamienna Góra (Landeshut) wurde im Jahr 2012 entdeckt (Duma - Rutka - Wojtucki 2014). Im Jahr 2015 wurden die archäologischen Forschungen fortgeführt. Ihr Ziel war vor allem die Erforschung eines hinter der eigentlichen Abdeckerei liegenden Raumes in unmittelbarer Nähe zum Galgen. Der Galgen bestand aus einer kreisförmigen, gemauerten Konstruktion mit einem Durchmesser von 5,2 m (Duma 2015, 76-77; Wojtucki 2009, 316-317). In seiner Nähe befand sich auch ein großes unbewegliches Objekt (Nr. 1/13). Parallel zu den archäologischen Forschungen wurden
Recherchen angestellt mit dem Ziel, alles Wissenswerte über die ehemaligen Hinrichtungsstätten und die Abdeckereien der näheren Umgebung zu sammeln.

Aus der Analyse der verfügbaren Quellen wissen wir, dass in Kamienna Góra (Landeshut) viele Todesurteile an der Hinrichtungsstätte vollstreckt wurden. Leider gibt es keine Gerichtsbücher über die Befragung der Angeklagten, die Urteile und Informationen zum weiteren Verfahren. Bei der Untersuchung wurden nur die in den Chroniken (Hayn 1825; 1845). enthaltenen Informationen und die wenigen Urteile des Appellationsgerichts zur Prag benutzt (Křepelková 1964, inw. 184-280). Die ersten bestätigten Hinrichtungen in Kamienna Góra (Landeshut) fanden erst in der zweiten Hälfte des 17. Jahrhunderts statt, obwohl die erforschte Hinrichtungsstätte nachweislich älteren Ursprungs ist. 1655 wurde ein unbekannter Mann durch Erhängen hingerichtet (Wojtucki 2009, 316-317). Ein Jahr zuvor wurde hier ein Selbstmörder begraben (Wojtucki 2009, 316). Die meisten Informationen über die ausgeführten Urteile an dieser Hinrichtungsstätte stammen aus dem 18. Jahrhundert. Wir finden sie sowohl in den Chroniken von Kamienna Góra (Landeshut) als auch in den Akten des Appellationsgerichts zur Prag (Wojtucki 2012). Die Quellen nennen leider kein Datum für die Errichtung des gemauerten Galgens, deren Fundamente im Zuge der archäologischen Forschungen entdeckt wurden (Duma - Wojtucki 2012, 12-21; Duma - Rutka - Wojtucki 2014, 10-23). Man vermutet, dass dies im 16. Jh. geschah, zu einer Zeit also, wo man die alten Holzobjekte gewöhnlich durch gemauerte Konstruktionen ersetzte. Ein Chronist aus dem in der Nähe liegenden Chełmsko Śląskie (Schömberg), erwähnte im Jahr 1593 beim Bau des Galgens in Chełmsko, dass man sich beim Bau des Galgens an dem von Kamienna Góra (Landeshut) orientierte. Er schrieb auch, dass er schon seit längerer Zeit gestanden habe (Lutterotti 1927). Es ist nachgewiesen, dass gegen Ende des 18. Jhs. und auch das ganze 19. Jahrhundert hindurch, der Scharfrichter aus Kamienna Góra (Landeshut) sich mit dem Abziehen der Kadaver beschäftigte. Aus der Mitte des 18. Jh. stammt ein Verzeichnis, das belegt, dass der Stadt damals 21 in der Nähe liegende Orte unterstellt waren. Noch früher waren es sogar 27, aber 6 von ihnen wurden der Scharfrichterei und Abdeckerei in Bolków (Bolkenhain) zugeschlagen (APJG 840, 76v; APJG 214, 61). Bis heute hat sich die Taxe aus dem Jahr 1649 für den Meister Gottfried Altvater erhalten.

Nachdem der Galgen in Kamienna Góra (Landeshut) nicht mehr gebraucht wurde, wurde er nicht vollständig abgebaut. Im Jahr 1820 entschied man sich, lediglich seine Säulen zu entfernen und die Konstruktion des zylindrischen Brunnens zwei Fuß, also etwa $60 \mathrm{~cm}$, abzusenken. Darauf wurde eine hölzerne Plattform gesetzt, auf der die Urteile ausgeführt werden sollten (APJG 214, 61). Nach diesem Umbau wurde ein gemauerter Rabenstein errichtet. Ab jetzt sollte man hier die Enthauptungen mit dem Beil ausführen. Rechtsgrundlage dafür war eine Verordnung aus dem Jahr 1811, nach der fortan alle Enthauptungen mit diesem Werkzeug erfolgen sollten (o. A. 1911, 164).

Die Tatsache, dass die Abdeckerei in unmittelbarer Nähe zum 


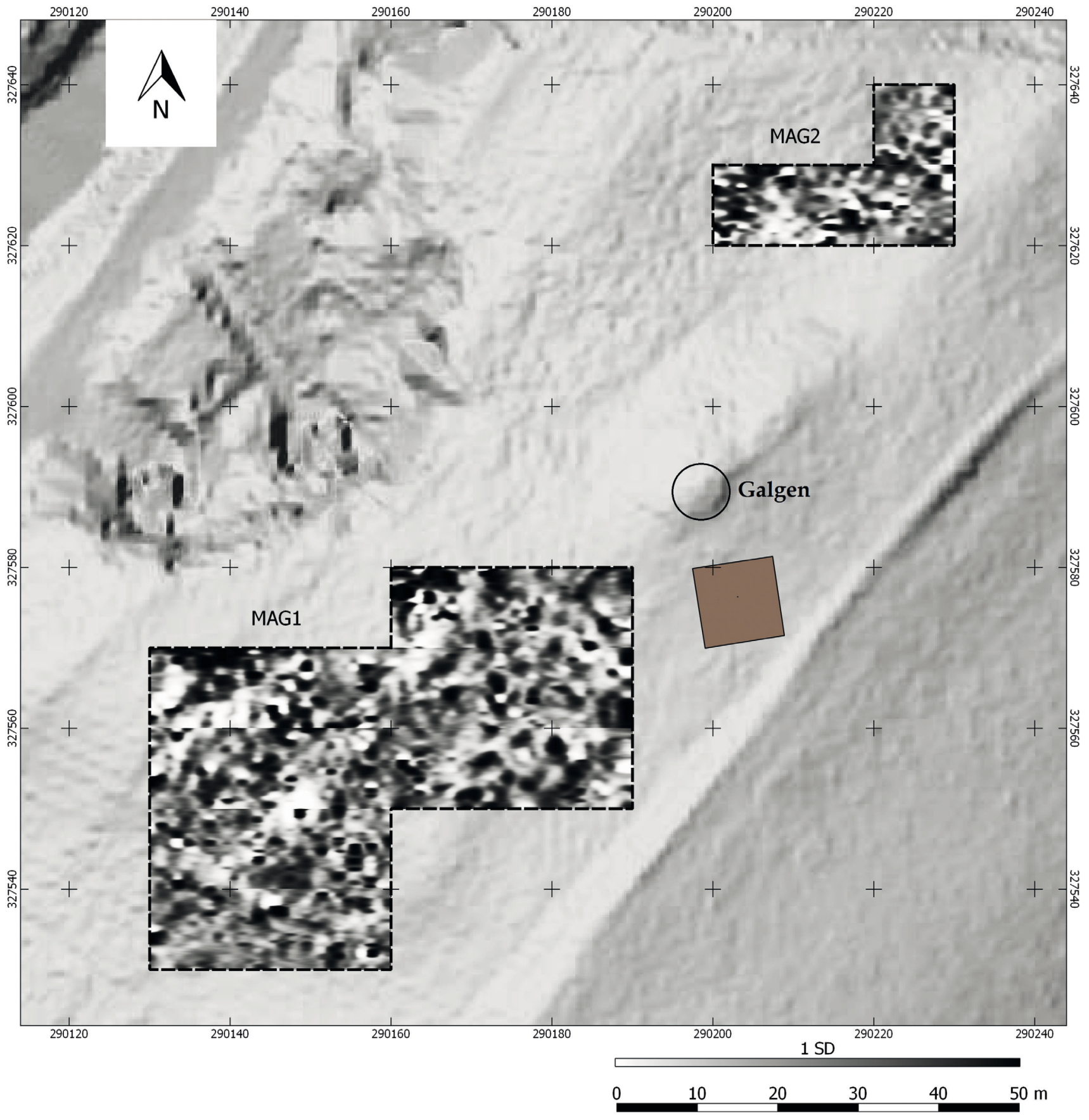

Figur 2. Kamienna Góra/Landeshut. Grabungsplan mit Hauptgrabungsschnitt mit Resten des gemauerten Galgens und Ergebnissen nichtinvasiver Prospektion. Bearbeitung: M. Mackiewicz

Galgen lag, war unter archäologischen Gesichtspunkten lange Zeit umstritten (Duma - Wojtucki 2015). Die Ausgrabungen in Kamienna Góra umfassten den eigentlichen Galgen und den davor liegenden Bereich, ein spürbar abgeflachtes Gelände. Zusätzlich wurden 14 Sondierungsforschungen durchgeführt mit dem Ziel, möglichst viel über die alte Abdeckerei zu erfahren. Es wurden auch nicht-invasive Forschungen von Maksym Mackiewicz und Bartosz Myślecki durchgeführt, konkret wurden so 26a $\left(1 \mathrm{a}=100 \mathrm{~m}^{2}\right)$ erforscht (Fig. 2). In der Humusschicht wurden zahlreiche Knochen, hauptsächlich Tierknochen, gefunden. Überraschend für die Forscher war aber die Tatsache, dass trotz der Überlieferung, dass Hinrichtungen auf dem beschriebenen Platz vollzogen und die Körper der Verurteilten direkt am Galgen verscharrt wurden sein sollen, keine menschlichen Spuren in den Gräbern gefunden wurden (Fig. 3). 2 Meter südlich der Galgenfragmente wurde ein großes, ovales Objekt mit einer Länge von bis zu 3 Meter und einer Breite von 2 Meter untersucht (Nr. 1/13). Es war mehr als 1 Meter im Boden versenkt. Im Inneren entdeckte man hunderte von Tierknochen, Schutt und 


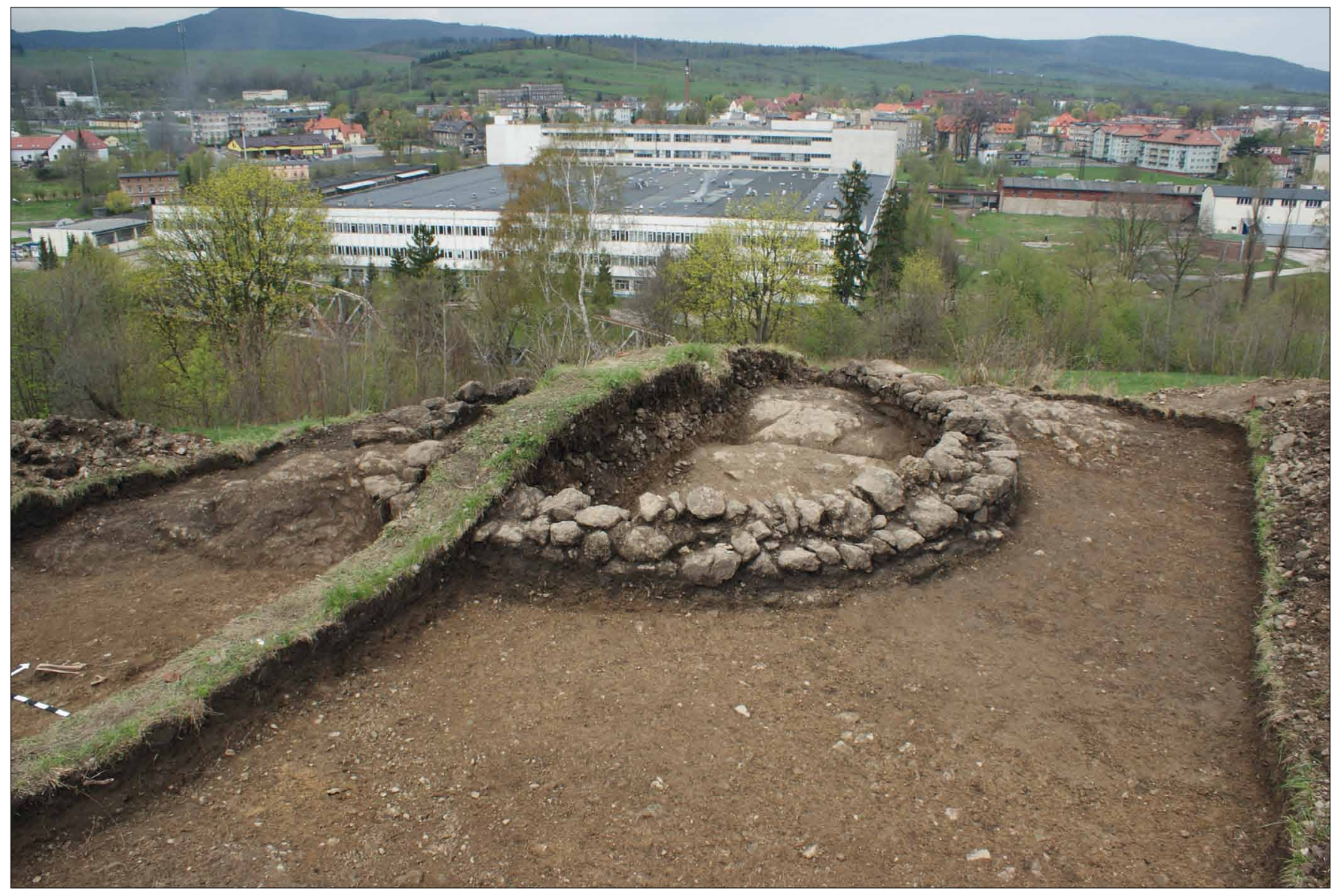

Figur 3. Kamienna Góra/Landeshut. Galgenreste und Stadt. Fot. P. Duma

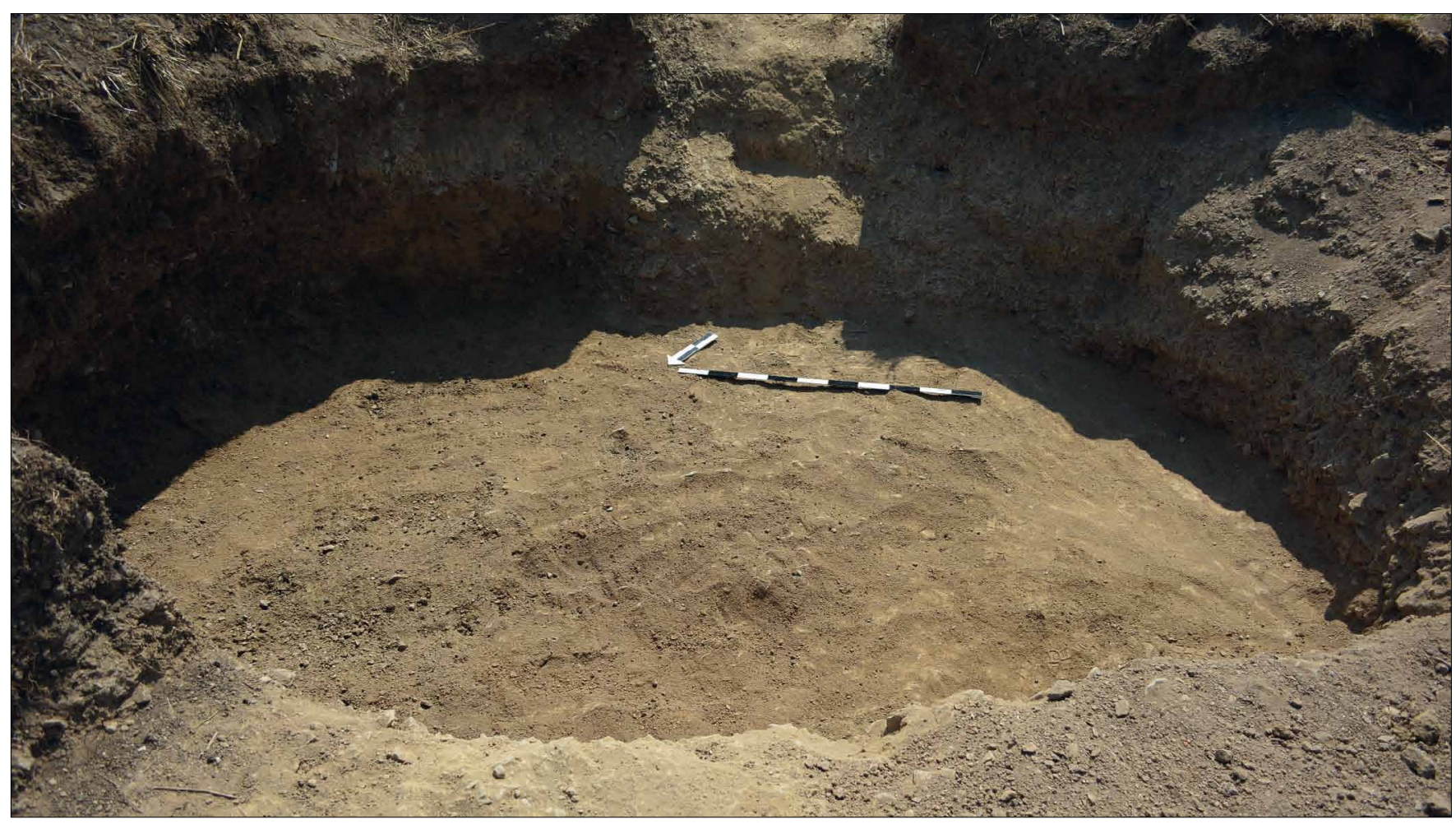

Figur 4. Kamienna Góra/Landeshut. Fundgrube (Schindergrube) beim Galgen, die mit großen Mengen von Tierknochen gefüllt war. Fot. P. Duma 


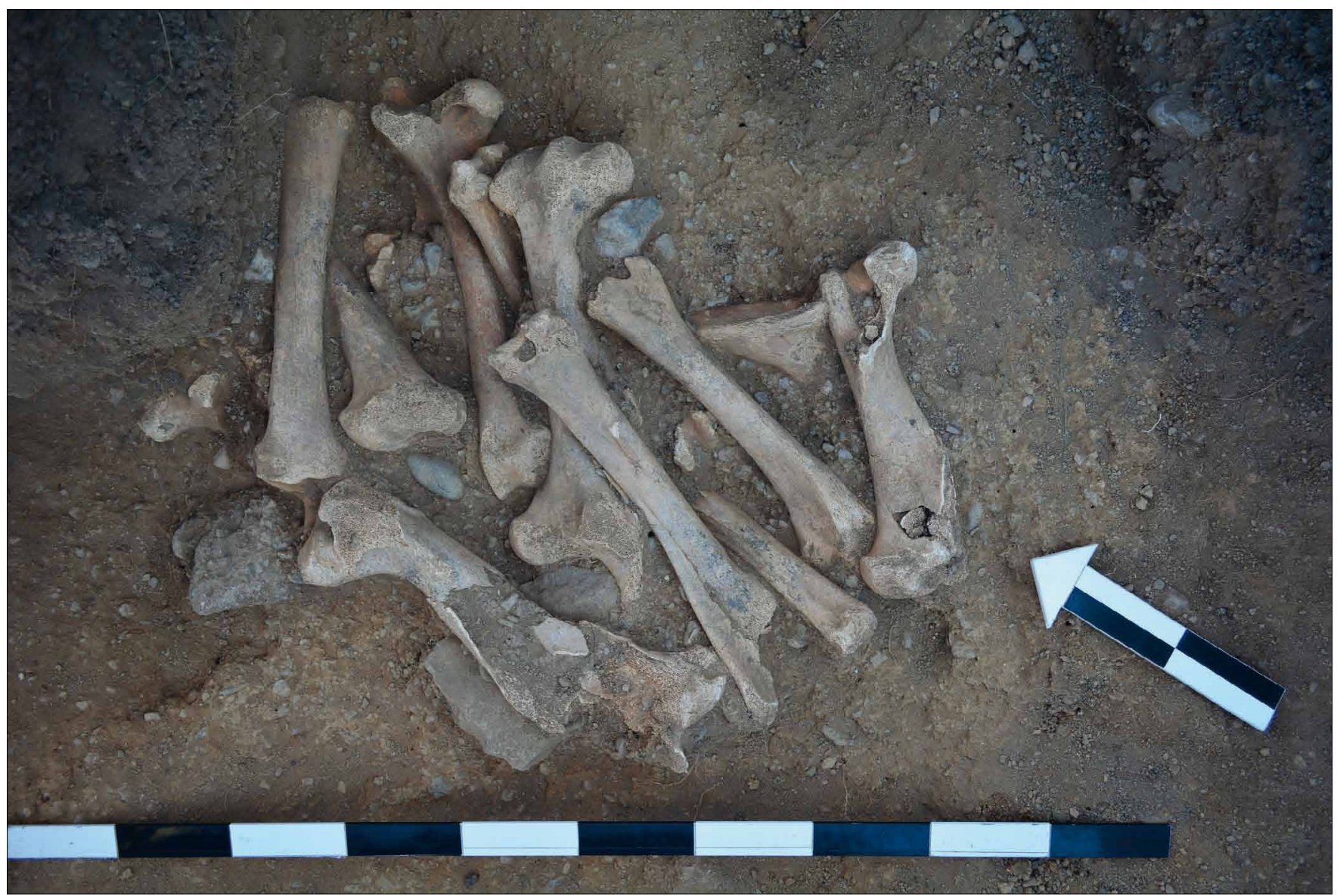

Figur 5. Kamienna Góra/Landeshut. Zweite Fundgrube in der Nähe des Galgens mit Tierknochen gefüllt. Fot. P. Duma

Steine, die wahrscheinlich vom gemauerten Galgen stammen (Fig. 4). Sie waren mit Mörtel bedeckt. Inmitten befanden sich zahlreiche Gegenstände, wie Keramik, Glas und Pfeifenfragmente. Die jüngsten von ihnen stammen aus dem frühen 19. Jahrhundert. Die Tierknochen waren stark zersplittert bzw. zerstört. Viele von ihnen waren verkohlt, was beweist, dass einige Tiere auf diesem Platz durch Verbrennen entsorgt wurden. Unter dem Material befanden sich auch wenige menschliche Knochen (Zähne, Finger, Wirbel). Am Fuße des Galgens, in der Sondierung-Forschung Nr. 12 entdeckte man auch ziemlich kleine Objekte mit einem Durchmesser von einem Meter (Nr. 3/15) und Viehknochen (Fig. 5). Die Gesamtfläche der Forschungen umfasste $170 \mathrm{~m}^{2}$. Die größte Grabung mit $10 \times 10 \mathrm{~m}$ wurde in direkter Nachbarschaft zum Galgen durchgeführt. Innerhalb der anderen Sondierungsgrabungen, die sich unter der Böschung befanden, fand man zahlreiche Tierknochen (einige davon verbrannt), die teilweise vom oberen Teil der Hinrichtungsstätte dorthin gelangten (sie waren mit Humus bedeckt). Schlagkräftigster Beweis, dass die alte Abdeckerei weit über den Bereich des steinernen Galgens hinaus reichte, sind zahlreiche Tierknochen, die in einer Entfernung von 30 Metern vom Galgen gefunden wurden.

Im Zuge der archäologischen Grabungen, die in drei Abschnitten am alten Hinrichtungsplatz und der Abdeckerei durchgeführt wurden, fand man zusammen 3.383 Tierknochen, die von Maciej Janeczek und Aleksander Chrószcz analysiert wurden. Aus diesem Material konnte man 1.036 Überreste qualitativ identifizieren. Das sind 30,6\% der ganzen Sammlung. Die Mehrzahl der Überreste stammte aus dem Objekt (Nr. 1/13) in der Nähe des Galgens. Im untersuchten Material dominierten Überreste von Pferdeskeletten (Equusferus f. caballus) und von Fleischfressern: Hunde (Canislupus f. familiaris) und Katzen (Felissilvestris f. domesticus). Bei den Rinderknochen (Bos primigenius f. taurus), waren die meisten Skelettfragmente mit dem Oberkörper (Fragmente von Rippe und Wirbel) verbunden. Gleichzeitig ist auffallend, dass sich (im Vergleich zum natürlichen Zerfall) vom Schädel und den oberen Extremitäten nur wenige Skelettfragmente erhalten haben. Leider erlauben die relativ wenigen Knochenüberreste von Haustieren, wie Kuh, Schaf, Ziege und Schwein keine exaktere Verwendung derselben. Im Fall der Pferde kann man mit Sicherheit annehmen, dass sie als Zugtiere genutzt wurden. Diese Nutzungsart war allerdings nicht so ausgeprägt, denn es gab keine größeren Spuren auf den erforschten Skelettfragmenten. Abgesehen von einigen Fällen festgestellter fortgeschrittener Malokklusion scheint es doch so zu sein, dass die Tiere von ihren Besitzern normal gehalten wurden. Archäozoologische Analysen haben gezeigt, dass sich diese 


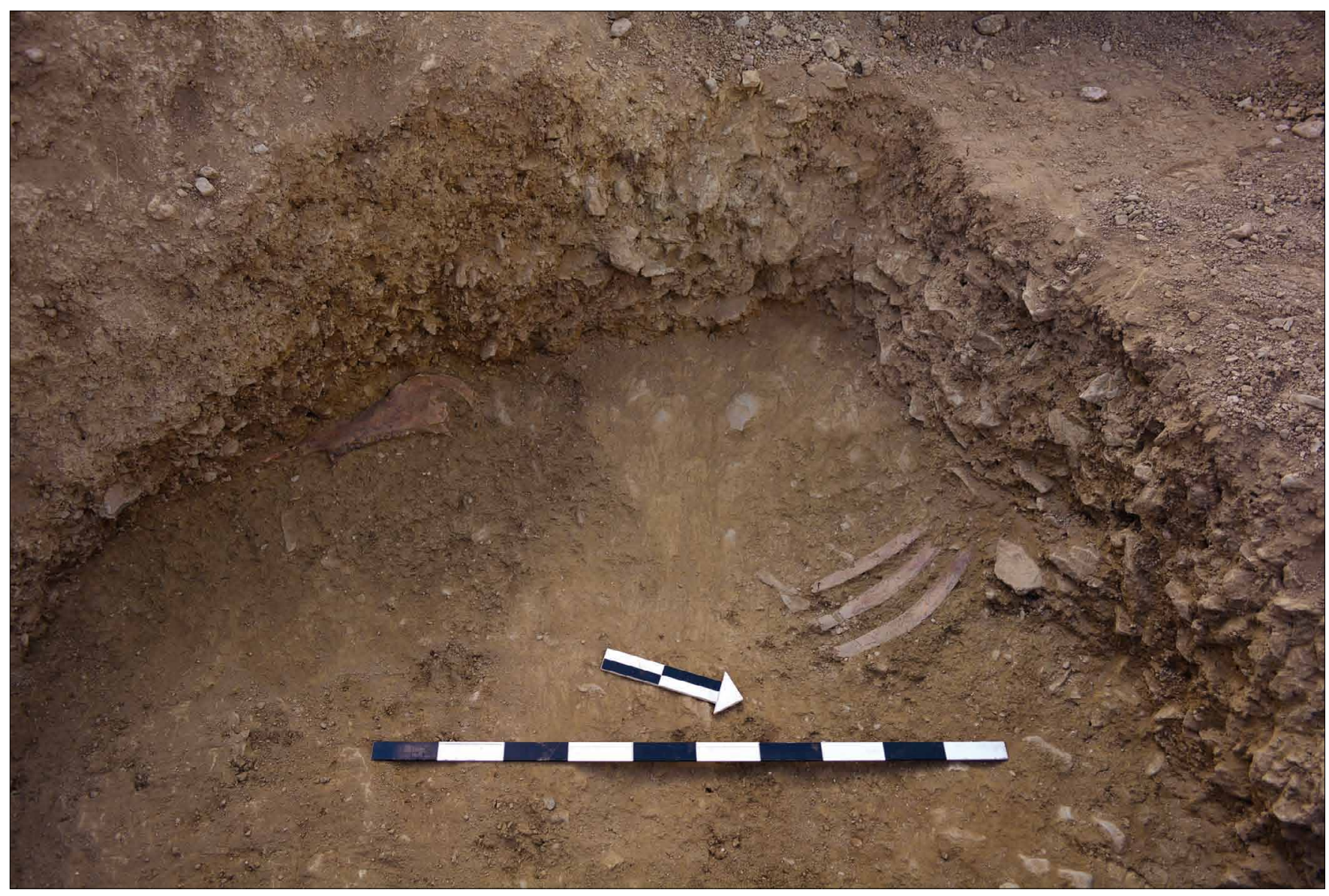

Figur 6. Kamienna Góra/Landeshut. Reste von Pferdeskeletten auf dem Grund der Fundgrube (Schindergrube). Fot. P. Duma

Sammlung an Tierresten von ähnlichen Sammlungen, die bei der Untersuchung von Siedlungsstandorten entdeckt wurden, unterscheidet. Es ist auch ein wertvolles Vergleichsmaterial für zukünftige Forschungen.

Es überrascht, dass in dem untersuchten Knochenmaterial nur wenige pathologische Knochenveränderungen gefunden wurden. Wir vermuten jedoch aufgrund verschiedener Berichte und Annahmen, die mit der Funktionsweise der ehemaligen Abdeckerei zusammenhängen, dass wir es hier mit kranken, für den Verzehr ungeeigneten Tieren, zu tun haben. Bei archäologischen Grabungen, die an der Hinrichtungsstätte durchgeführt wurden, wurden keine anatomischen Reste (weder vom Mensch noch vom Tier) gefunden. Trotzdem haben die Funde unser Wissen über das Funktionieren frühneuzeitlicher Abdeckereien bereichert. Zum ersten Mal in der Geschichte archäologischer Grabungen ist es gelungen, eine wirkliche Schindergrube (Nr. 1/13) auszuheben (Fig. 6). Vermutlich entsprach ihre Größe nicht den üblichen Gruben, die aus den Quellen bekannt sind und sich in der Nähe von Großstädten befanden, Nichtsdestotrotz enthielten sie sehr viel wertvolles Knochenmaterial.

Die Sammlung auch kleinster Funde ergänzt unser Wissen über die Abdeckerei und die Hinrichtungsstätte. Viele stammen aus jener Zeit, als die Hinrichtungsstätte ihre eigentliche
Funktion eingebüßt hat und dieses Gebiet landwirtschaftlich genutzt wurde. Andere Kleinfunde (insbesondere aus dem 17. und 18. Jh.) stammen zweifellos aus der Epoche, als sie noch nicht ihre Funktion verloren hatte. Die reichlich vorhandenen Luxuskeramikfragmente (u. a. Fragmente von Steinzeug) sind interessante „Abfallprodukte“ dieser markanten Stätte. Möglicherweise stammt ein Teil des Materials aus städtischen Abfällen, z. B. aus Kloaken, deren Hinterlassenschaften hierhergebracht wurden. Zweifellos gehören auch die vielen Kaolinpfeifen, deren Reste gesichtet wurden, den Scharfrichterknechten. Sie weideten die Tiere auf der Hinrichtungsstätte aus, vergruben und äscherten sie ein.

\section{DIE HINRICHTUNGSSTÄTTE UND DIE ABDECKEREI IN ZŁOTORYJA (GOLDBERG)}

Die zweite Hinrichtungsstätte mit angeschlossener Abdeckerei befindet sich auf Góra Mieszczańska in Złotoryja (Goldberg) und ist historisch gut dokumentiert. Die genaue Lokalisierung des Platzes, wo ursprünglichdie Urteile vollstreckt wurden, war möglich dank der Sondierungsforschungen, die im 2015 Jahr durchgeführt wurden. Die Grabungsstelle ergab sich aufgrund der Archivlandkarten und der Feldbeob- 


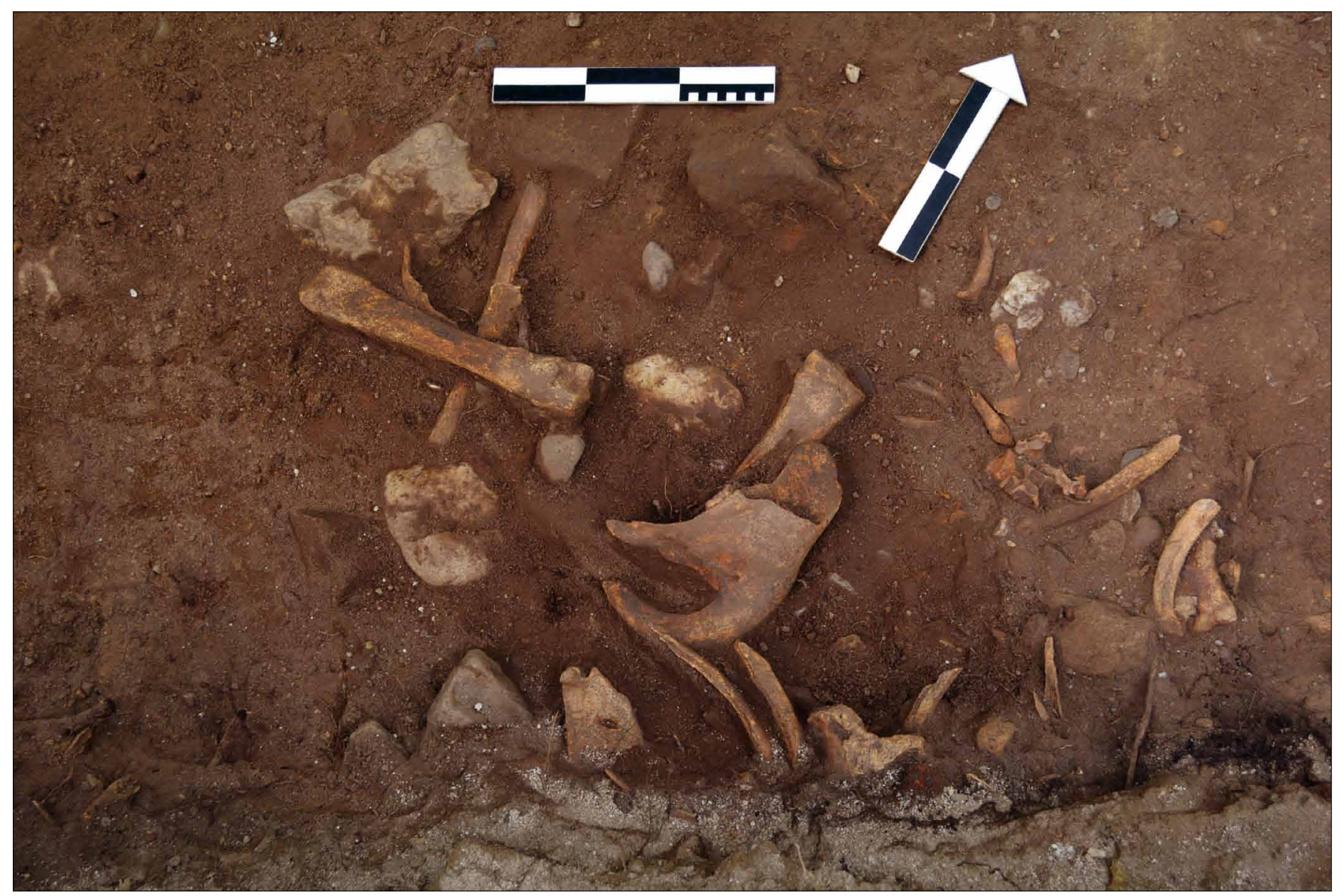

Figur 7. Złotoryja/Goldberg. Menschliche und tierische Knochen, die im Inneren des Galgens gefunden wurden. Fot. P. Duma

achtungen. Im Zuge der Sondierungsforschungen entdeckte man ein gut erhaltenes Fragment des Galgenfundaments und eine Schicht von dem Schutt, der mit dem Abriss des Galgens aus dem Juni 1810 zusammenhängt. Im Juli 2016 wurden die archäologischen Forschungen weitergeführt. Man fand gut erhaltene Fragmente des gemauerten Galgens und weitere Kulturhinterlassenschaften, die im Inneren der Konstruktion lagerten. Es wurden auch fünf Sondierungsgrabungen durchgeführt; Gräber von Verurteilten in der Nähe des Galgens wurden dabei nicht gefunden.

Die Analyse der erhaltenen Quellen geben Aufschluss über einige Hinrichtungen, die dort vollgezogen wurden. Die ersten Hinrichtungen fanden hier Ende des 15. Jh. statt. Das Gebiet wurde auf den Landkarten aus der Mitte des 18. Jh. als unheimlicher Mordt Grundt bezeichnet. Die Abdecker und Scharfrichter sind dort nachweislich über mehrere Jahrhunderte tätig gewesen. Entsprechende Namen kann man in verschiedenen Urkunden finden, zuletzt noch Anfang des 20 Jhs. Ein Mangel an entsprechenden Gräbern führte dazu, dass man die Leichen zum Tode Verurteilter und Selbstmörder unter dem Galgen begraben hat.

Aus den Quellen wissen wir auch, dass der Goldberger Scharfrichter nicht nur die Gerichtsurteile exekutieren musste, sondern auch für Ordnung im gesamten Weichbild (Stadt und Vororte) verantwortlich war. Zu seinen Aufgaben gehörten u. a. auch die Entfernung von Aas, das er im sogenannten Schindanger in unmittelbarer Nähe zum Hinrichtungsplatz, verlochte.

Das Ende des Galgens von Goldberg wurde im April des Jahres 1810 eingeläutet, als die Stadtbehörden kurzerhand entschieden, die Hinrichtungsstätte abzureißen. Die erste Eingabe in dieser Angelegenheit wurde im April 1810 mit der Begründung versehen, dass der Galgen, der auf dem Hügel rechterhand von Legnica stand, stark beschädigt sei und die Balken verfault (APW 317, 1-6) wären. Der gemauerte Galgen wurde abgerissen und die alte Hinrichtungsstätte in einen Stadtpark umgewandelt.

Wir vermuten, dass die in den Quellen genannten Gräber von Kriminellen und Selbstmörder in einiger Entfernung vom zentralen Platz gelegen haben. Es lassen sich zahlreiche menschliche und tierische Knochen nachweisen, die scheinbar ungeordnet verlocht wurden (Fig. 7). Diese wurden hauptsächlich bei der Erkundung von Schichten im mittleren Bereich des Galgens gefunden. Bei den Sondierungsgrabungen wurden nur wenige Knochen gefunden. Die Anzahl der menschlichen Knochen übersteigt bei weitem die Anzahl der dort gefundenen Tierreste. Diese Überreste stehen zweifellos im Zusammenhang mit der Abdeckerei, die sich in unmittel- 


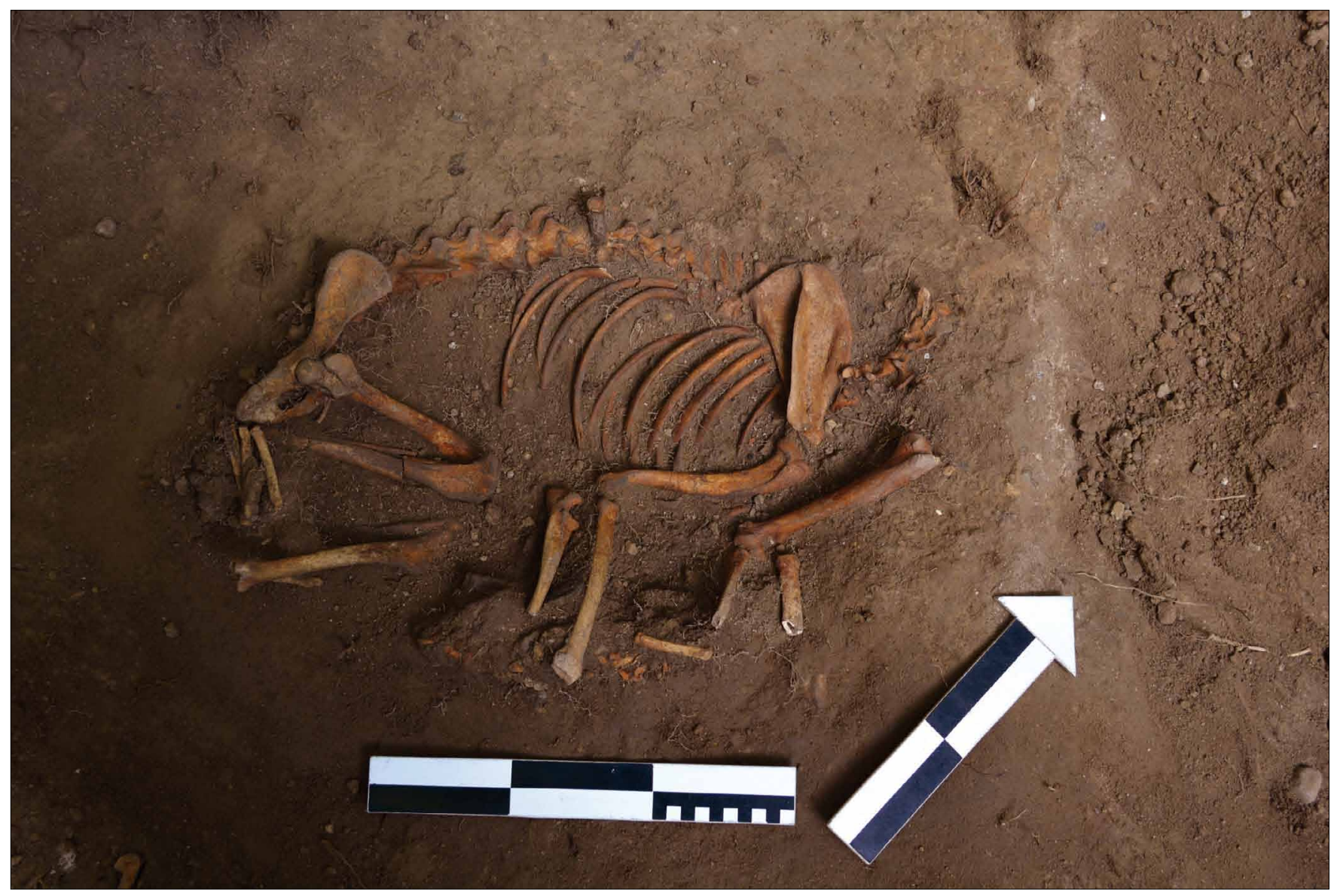

Figur 8. Złotoryja/Goldberg. Galgeninnensicht mit Hundeskeletten in situ. Fot. P. Duma

barer Nähe befand. Wir schließen daraus auch, dass einige Aktivitäten im Zusammenhang mit der Verarbeitung von Aas standen, die in unmittelbarer Nähe zum Galgen durchgeführt wurden, und sein Inneres diente gleichzeitig als Mülldeponie. Neben zahlreichen Knochenresten, fand man auch kleinere Objekte, die später besprochen werden sollen. Viele von ihnen können direkt mit dem Hinrichtungsakt verknüpft werden. In den erhaltenen Quellen finden wir kein Datum für die Errichtung des Galgens in Złotoryja (Goldberg). Aufgrund von Keramikfragmentfunden und einer Münze, die in den Schichten des Gebäudes (Sachsenpfennig aus dem Jahr 1541) lagen, gehen wir davon aus, dass der Goldenberger gemauerte Galgen schon im 15. Jahrhundert aufgestellt wurde und in späteren Jahrhunderten aufgefüllt worden ist.

Aufgrund der durchgeführten archäologischen Forschungen ist klar, dass das Innere des Galgens für das Ablegen von Tierresten aus einer nahe gelegenen Abdeckerei verwendet wurde, die in der Quelle erwähnt wird. Die Menge an Resten zeigt, dass sich die Bereiche Abdeckerei und Hinrichtungsstätte überschneiden. Das Fehlen von Tierbestattungen und Knochen in anderen Ausgrabungen weist indirekt darauf hin, dass Aas von den Mauern entfernt wurde. Die entdeckten menschlichen und tierischen Knochen entbehren jeglicher anatomischer Ordnung. Nur im nördlichen Teil der Grabung wurde neben dem Fundament das Skelett eines Hundes entdeckt (Fig. 8-9), während im zentralen Teil ein nahezu vollständiges Skelett einer Katze freigelegt werden konnte. Einige Tierknochen wurden verbrannt oder weisen Brandspuren nach. In vielen Fällen waren die menschlichen Knochen in einem schlechten Erhaltungszustand. Sie waren wahrscheinlich lange Zeit atmosphärischen Bedingungen ausgesetzt. Darüber hinaus wurden in der beschriebenen Schicht zahlreiche, mit der Vollstreckung der Todesurteile zusammenhängende Denkmäler gefunden. Dazu gehören Glieder von Eisenketten und Eisenhaken. Aus historischen Quellen wissen wir, dass ähnliche Haken zum Fertigen von Seilen oder Ketten verwendet wurden, bei denen Menschen an Holzbalken gehängt wurden. Im zentralen Galgen, nahe des Naturbodens, wurde eine vollständige Kette, entdeckt (Fig. 10).

Die Gesamtzahl der Knochen lag bei 8.989 Stück. Nur 5,65\% aus dieser Sammlung waren menschliche Knochen. Der Großteil stammt aus dem Inneren des Galgens und wurde dort vor 1810 deponiert. Die erforschten Lagen waren mit einer Schuttschicht $(15 \mathrm{~cm})$ bedeckt, die während des Abbaus des Galgens entstand. Wenige Knochen wurden außerhalb des Fundaments gefunden. 


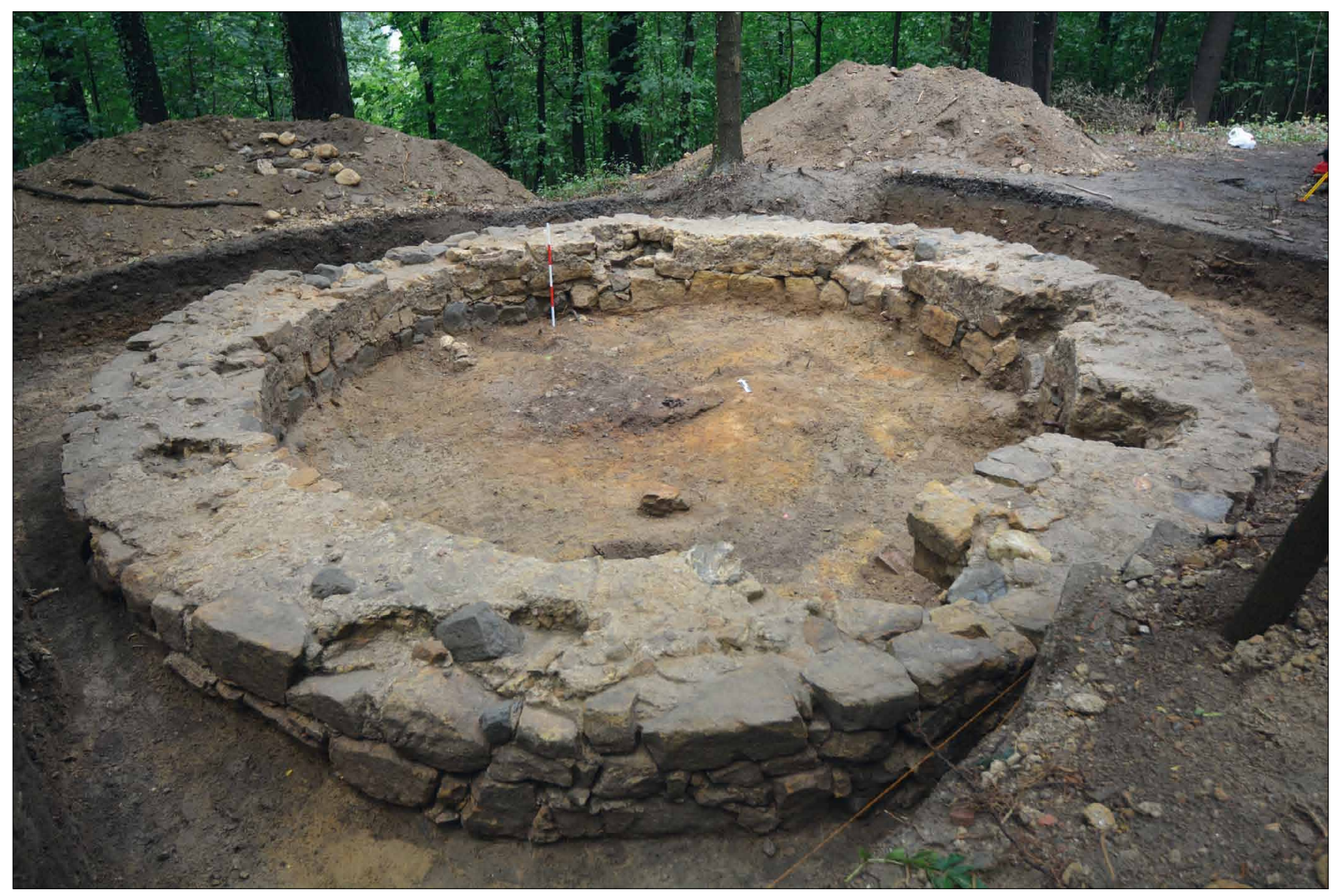

Figur 9. Złotoryja/Goldberg. Ansicht in nördlicher Richtung nach Galgenfundamentresten. Fot. P. Duma

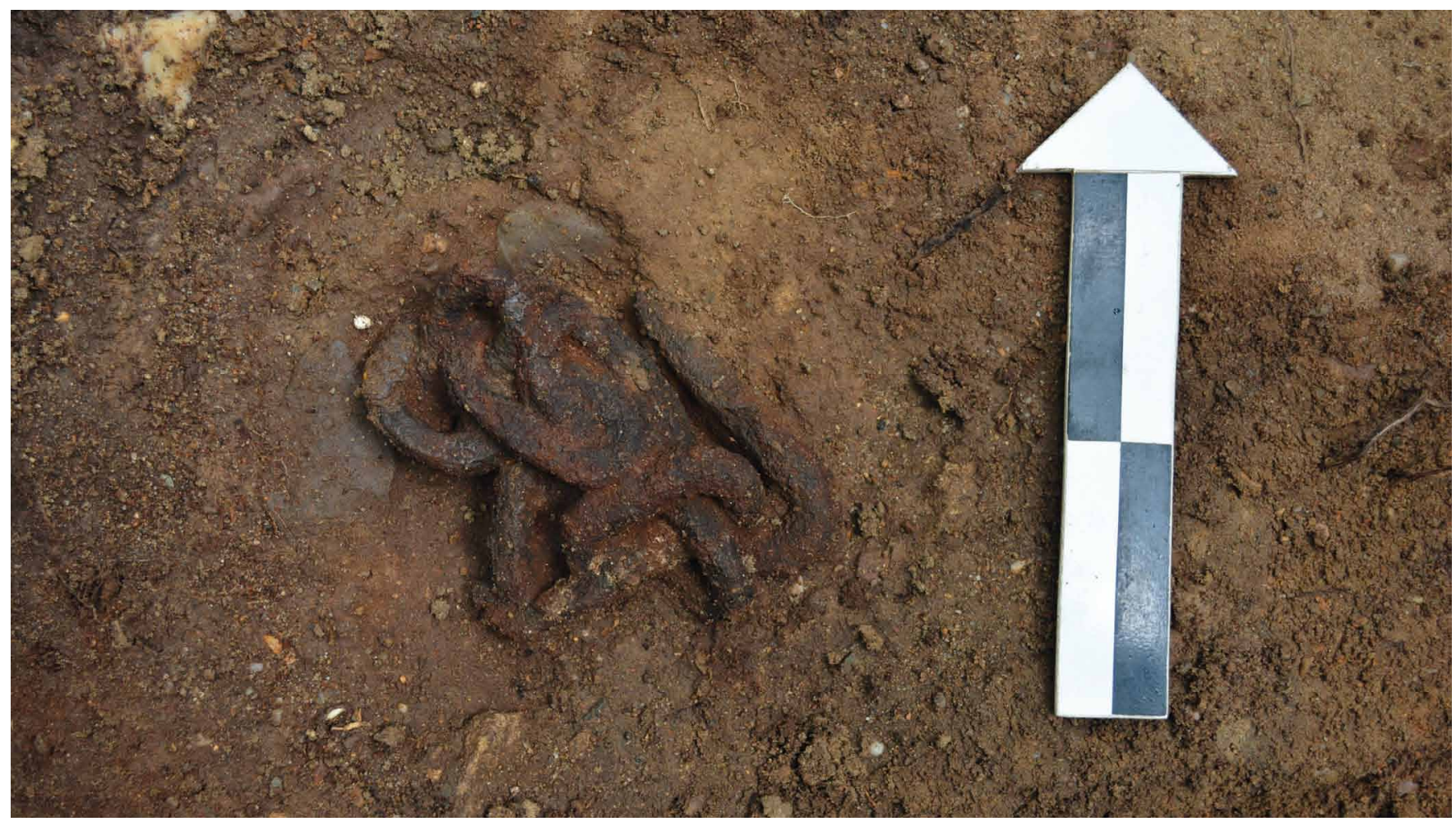

Figur 10. Złotoryja/Goldberg. Im Galgensinneren gefundene Exekutionskette in situ. Fot. P. Duma 


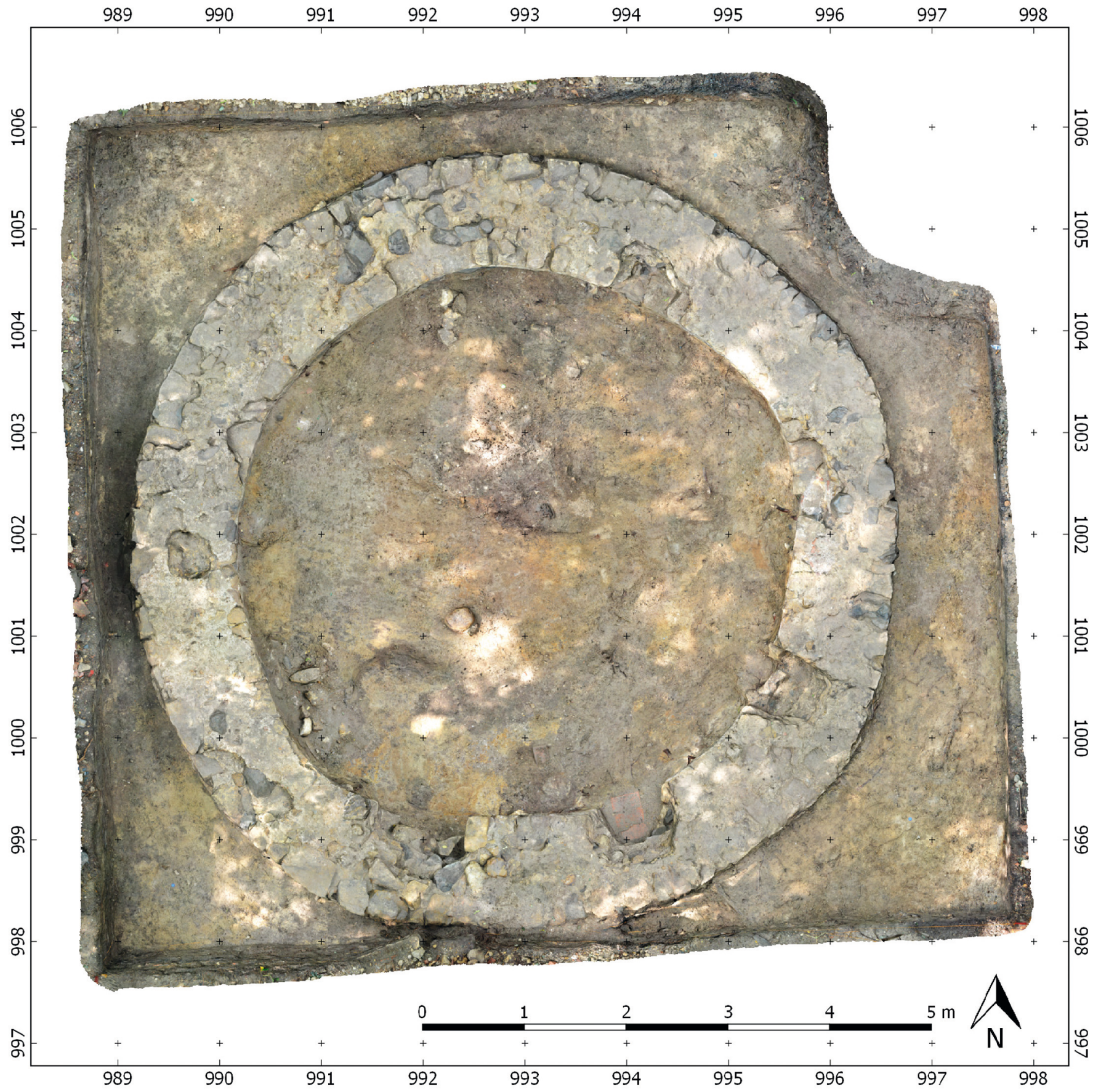

Figur 11. Złotoryja/Goldberg. Galgensfundamente, Photogrammetrie nach Beendigung der Grabungen. Bearbeitung. M. Mackiewicz.

Wie die Analyse zeigte, stammten 40,97\% des Knochenmaterials hauptsächlich von Haustieren. Der geringe Prozentsatz artenspezifischer Knochenreste ist sowohl auf die Art der Befunde als auch auf den Zustand ihrer Erhaltung zurückzuführen. In beiden Fällen dominierten eindeutig Skelettüberreste von Pferden (Equusferus f. Caballus) und Hunden (Canislupusf. Familiaris). Die verbleibenden Haussäugetiere übertrafen nicht den Prozentsatz von 15,97\%, und Wildtiere stellten keinen bedeutenden Teil der Sammlung dar. Die erhaltenen Ergebnisse zeigen eindeutig, dass es sich bei dem untersuchten Material nicht um Knochenreste handelte, die nach dem Verzehr von Fleisch von Zucht- und Wildtieren übriggeblieben waren. Bei den Überresten von Pferden, wurde ein signifikanter Überschuss an Skeletteilen des Rumpfes beobachtet.

Bei der anthropologischen Analyse anderer menschlicher Knochen gab es große Probleme wegen des schlechten Erhaltungszustandes. In einem Fall konnte anhand des Er- 
haltungszustandes der Zähne, das Alter der Person auf ungefähr 50 Jahre geschätzt werden. Bei der Erkundung der inneren Schichten des Galgens konnten keine anatomischen Reste zugeordnet werden, da menschliche Knochen chaotisch mit Tierknochen vermischt wurden und keine Anhäufung bildeten, was darauf schließen lässt, dass sie aufgrund unterschiedlicher Handlungen eingebracht und dann mehrfach wieder verschoben wurden. In unmittelbarer Nähe der Fundamente des Galgens wurden einzelne, kleine Knochenreste gefunden. Insgesamt wurden 193 menschliche Knochen erkannt und beschrieben, darunter auch Knochen von mindestens drei erwachsenen Männern.

Die Fundamente des Galgens zählen heute zu den am besten erhaltenen Überresten ehemaliger Galgen, die in Niederschlesien je ausgegraben wurden (Fig. 11). Das Fundament befindet sich von der Westseite her $71 \mathrm{~cm}$ unter dem Boden. Die Breite des Fundaments im nördlichen Teil betrug $102 \mathrm{~cm}$ und im westlichen Teil $114 \mathrm{~cm}$. Der Außendurchmesser des Fundaments auf der Ost-West-Linie war 7,65 m, der innere 5,37 m. Die beiden Hinrichtungsstätten machen deutlich,wie wichtig Fragen im Zusammenhang städtischer Hygiene machen. Tierisches Knochenmaterial, das in beiden Fällen gegenüber menschlichen Knochen überwiegt zeigt, dass die häufigste Nutzung beider Plätze mit der Abdeckerei einherging. Knochenmaterial von Hinrichtungen, die dort quellenmäßig belegt und durchgeführt wurden, fehlen oftmals. Wir müssen bedenken, dass Leichen Verurteilter, oft bis zur vollständigen Verwesung zur Schau gestellt wurdenund damit keine Spuren hinterlassen haben und wenn ja, nur sehr eingeschränkt. Auch wurden bei den beiden Grabungen, trotz des breitangelegten Forschungsvorhabens keine anatomischen Gräber gefunden. Der Grad der Fragmentierung und Zerstreuung der Tierreste lässt nur den einen Schluss zu, dass die Tiere nach dem Zerlegen nicht begraben wurden. Sie lagen eher an der Oberfläche, wo das Gewebe vollständig zersetzt wurde und wurden dann verbrannt oder ins Innere des Galgens geworfen, wie es beim Galgen in Złotoryja der Fall war oder wurden in der Schindergrube vergraben. So war es in Kamienna Góra. Die Aktivitäten wechselten immer mal wieder und unterliegen zeitlichen Bedingungen, die jedoch bei der Erforschung schwer erkennbarwaren. Die Annahme, dass die meisten Hinrichtungsstätten nur für Humanexekutionen gedacht waren, erfährt insofern eine Modifizierung, als nunmehr die Sanitärfunktion eine übergeordnete Rolle einnimmt und durch entsprechendes Material belegt werden kann. Diese Erkenntnis deckt sich mit anderen Hinrichtungsstätten, die in den letzten Jahren durch die Autoren erforscht worden sind. Es gibt aber auch solche, wo überhaupt keine Tierreste gefunden wurden. Daher kann dieses "Modell“ nicht auf alle schlesischen Städten gleichermaßen übertragen werden, sondern entspricht analogen Positionen aus anderen europäischen Gebieten (Manser 1992; Genesis 2014, 55-57).

Übersetzung: Karolina Wojtucka, Korrektur: Helmut Belthle

\section{QUELLEN}

APW (317): Archiwum Państwowe we Wrocławiu, Oddział w Legnicy, Akta miasta Złotoryi, sygn. 317, Akten des Magistrats zu Goldberg in Schlesien betreffend den Bürgerberg und die Unterhaltung der Anlagen desselben.

APJG (214): Archiwum Państwowe we Wrocławiu, Oddział w Jeleniej Górze, Akta miasta Kamiennej Góry, sygn. 214, Acta der StadtverordnetenVersammlungzuLandeshutvomJahre 1820.

APJG (840): Archiwum Państwowe we Wrocławiu, Oddział w Jeleniej Górze, Akta miasta Kamiennej Góry, sygn. 840, Acta Generalia des Magistrats zu Landeshut betreffend die hiesige Scharfrichterei deren Abdeckereibetrieb und Verpflichtungen Erfühigung, Vol. III.

\section{LITERATUR}

Duma, Paweł (2015): Śmierć nieczysta na Ślasku. Studia nad obrzadkiem pogrzebowym społeczeństwa przedindustrialnego, Wrocław, Uniwersytet Wrocławski.

Duma, Paweł - Rutka, Honorata - Wojtucki, Daniel (2014): Badania archeologiczne byłego miejsca straceń w Kamiennej Górze w 2013 r. Pomniki Dawnego Prawa, 25, 10-23.

Duma, Paweł - Wojtucki Daniel (2012): Badania sondażowe na dawnym miejscu straceń w Kamiennej Górze w październiku 2012 roku. Pomniki Dawnego Prawa, 20, 12-21.

Duma, Paweł - Wojtucki, Daniel (2015): Miejsce straceń i zaplecze rakarskie w śląskim mieście wczesnonowożytnym, In: Korpalska, W. - Ślusarczyk W. (Hrsg.): Czystość i brud. Higiena nowożytna (XVIII-XIX w.), Bydgoszcz, Collegium Medicum, 255-270.

Genesis, Marita (2014): „Das Gericht” in Alkersleben- archäologischer und historischer Nachweis einer mittelalterlichen Richtstätte in Thüringen unter Hinzuziehung anthropologischer Analysen, Langenweissbach, Beier \& Beran.

Hayn, C.F.W. (1825): Denkwürdigkeiten Landeshuts und einige benachbarter Orte, Landeshut.

Hayn, C. F. W. (1845): Chronologische Notizen oder merkwürdige Begebenheiten die sich in der Vorzeit und Gegenwart in und um Landeshut ereignet haben, Landeshut.

Křepelková, Anita (1964): Apelační soud 1548-1783, Praha.

Lutterotti, Nikolaus (1927): Wie die Schömberger anno 1692 ihr Hochgericht erneuerten. Volksblatt aus 1. 12. 1927.

Manser, Jörg(1992): Richtstätte und Wasenplatz in Emmenbrücke (16.-19. Jahrhundert), 1-2, Basel.

Ohne Autor (1911): Das Jubiläum des preußischen Richtbeiles. Zittauer Stimmen, Blätter für heimatliche Geschichte, Beiblatt, 5 (41), 164.

Wojtucki, Daniel (2009): Publiczne miejsca straceń na Dolnym Śląsku od XV do połowy XIX w., Katowice, Fundacja Zamek Chudów.

Wojtucki, Daniel (2012): Korespondencja Praskiej Izby Apelacyjnej z miastami na Śląsku w latach 1548-1740. Klio. Czasopismo poświęcone dziejom Polski i powszechnej, 23 (4), 167-176.

\section{AUTOREN}

Dr. Duma, Pawel, (geb. 1984), Archäologe, email: pawelduma84@ gmail.com, Arbeitsschwerpunkte: Rechtsarchäologie, Tod und Begräbnisstätten in Mittelalter und Früher Neuzeit, Historische Archäologie, Stadt- und Siedlungsarchäologie

Dr. Wojtucki, Daniel, (geb. 1977), Historiker und Archivar, email: daniel.wojtucki@centrum.cz, Arbeitsschwerpunkte: Scharfrichteramt in Schlesien und der Oberlausitz (16.-19. Jahrhundert), Rechtsarchäologie, mittelalterliche und neuzeitliche Geschichte Schlesiens, Militärgeschichte 